\title{
LA NARRACIÓN AUTOBIOGRÁFICA COMO ELEMENTO ESCENOGRÁFICO EN LA INTERPRETACIÓN DE LA OBRA DE ARTE
}

\author{
THE AUTOBIOGRAPHIC NARRATION AS A SCENARIO \\ ELEMENT IN THE INTERPRETATION OF THE ARTWORK
}

\author{
Soledad Fernández Inglés \\ (Universidad de Málaga, España) \\ sfernandezingles@gmail.com
}

Recibido: 27 de octubre 2020 / Aceptado: 8 de febrero 2021

Resumen. La autobiografía como narración utilizada en la mediación cultural permite la comprensión del patrimonio intercultural, activar la alfabetización crítica y crear un sentido de pertenencia a un lugar. Este artículo recoge cómo utilizan la narración autobiográfica los servicios educativos de la Galleria d'Arte Moderna e Contemporanea de Bérgamo (GAMeC), en coherencia al desarrollo de su política de inclusión social y cultural.

La búsqueda de una metodología adecuada, que acercara su colección permanente a visitantes procedentes de otros contextos culturales y geográficos, les condujo en 2014 a idear y poner en marcha un proyecto que abarcaba memoria y tradición (patrimonio cultural inmaterial) para preservar la identidad cultural. De este modo, salvaguardaba la función transmisora y comunicadora del museo y, al mismo tiempo, ayudaría a sentirse parte de a una población que, como individuo, forma parte de la sociedad que la ha acogido. Para llegar a ello, crearon puentes utilizando el diálogo entre la obra de arte y la vida cotidiana del narrador, pero sin perder de vista, en los contenidos que fueron creando, los objetivos: formar, experimentar y cohesionar.

No han faltado las críticas por parte de algunos comisarios, que consideran esta iniciativa carente de rigor científico por su elevada dosis de subjetividad, olvidando que se trata de una herramienta para acercar el arte a quienes desconocen los códigos para descifrar e interpretar algunas obras de arte.

Palabras clave: Narración autobiográfica, museología crítica, pedagogía crítica, patrimonio cultural, mediación cultural.

Autobiography as a narrative used in cultural mediation allows for the understanding of intercultural heritage, activating critical literacy and creating a sense of belonging to a place. 
This article shows how the educational services of the galleria d'Arte Moderna e Contemporanea de Bergamo (GAMeC) use autobiographical narration, in coherence with the development of its policy of social and cultural inclusion.

The search for a suitable methodology to bring the culture that emerges from its permanent collection closer to visitors from other cultural and geographical contexts led them in 2014 to devise and implement a project that encompassed memory and tradition (intangible cultural heritage) to preserve cultural identity. In this way, it safeguarded the museum's transmitting and communicating and, at the same time, help function would be safeguarded and, at the same time, it would helped to feel part of a population that, as an individual, is part of the society that has welcome it. To achieve this, they created bridges by using the dialogue between the work of art and the everyday life of the narrator, but without losing sight, in the contents they created, of the objectives: to train, experiment an unite.

There has been no lack of criticism from some curators, who consider this initiative to lack scientific rigor due to its high dose of subjectivity, without understanding that it is a tool to bring art closer to those who do not know the codes to decipher and interpret some works of art.

Keywords: Autobiographical narration, critical museology, critical pedagogy, cultural heritage, cultural mediation.

\section{Introducción a las narrativas y tendencias de la educación museal}

La atención que la nueva museología presta a la relación entre la persona y la cultura material e inmaterial pone de manifiesto que la potencialidad de los bienes culturales puede transformar a un público pasivo del museo en un agente activo del patrimonio. El museo, espacio vivo en el que se verifica un encuentro entre artistas de todos los tiempos, se convierte en un lugar para reflexionar. Por ello, en las últimas décadas, los museos han ido consolidando su posición dentro del ámbito educativo como espacios legítimos de aprendizaje.

Los museos de arte en el sentido moderno nacieron a finales del siglo XIX principios del XX. Cuando se fueron enriqueciendo con nuevas funciones, pasaron de ser un contenedor y conservador de objetos a productor de cultura. Ello dio pie a dos narrativas y a dos tendencias en educación museística: la narrativa formalista o estética -cuyo objetivo 
educativo es la apreciación estética y el desarrollo del buen gusto- y la narrativa disciplinaria, que tiene como fin la instrucción.

Durante la segunda y tercera década del siglo XX inicia la narrativa experiencial. Educadores como Dewey o María Montessori vieron la potencialidad de los museos para un aprendizaje artístico no estructurado, influyendo con sus ideas en algunos responsables de departamentos de educación estadounidenses atraídos por las propuestas de 'aprender haciendo', y el uso de nuevas metodologías basadas en el diálogo (David, 1946, citado en Zeller, 1989, p. 55). Son los años de la reforma social llevada a cabo por Roosevelt, de los debates sobre la cultura de la Escuela de Frankfurt fundada en 1923 y de las propuestas de la investigación sobre la psicología del aprendizaje. El museo empieza a interesarse sobre cómo aprende el visitante y, en consecuencia, a desarrollar proyectos para hacer eficaz la misión educadora.

El siguiente y natural paso fue pensarlo como instrumento para el cambio social: el museo ya no podía tener solo una misión estética y otra disciplinaria, sino que era necesaria la tercera, la misión social. No obstante, seguirá trabajando bajo los aspectos estéticos y disciplinares hasta que en los Estados Unidos y la Gran Bretaña de los setenta -y fruto del destilado de los cambios políticos, económicos y filosóficos de los años sesenta- empieza a ser apreciable su transformación. Son los años en los que emergen los primeros departamentos de educación europeos.

A partir de la década de los ochenta, el museo inicia a plantearse cuestiones ligadas a la accesibilidad física, social, económica e intelectual surgiendo una nueva narrativa, definida por Carla Padró (2005) comunicativa o de acceso. Aunque se trata de una narrativa nacida en los años treinta del pasado siglo, hay que esperar más de medio siglo para que adquiera relevancia. Cambia el concepto de visitante, que ahora se considera constructor del conocimiento, por lo que los nuevos proyectos educativos buscan negociar otros significados huyendo de cualquier imposición. Para lograrlo, se tienen en cuenta las teorías constructivistas de aprendizaje, asumiendo que los visitantes no construyen las mismas asociaciones partiendo de análogas preguntas, porque cada uno tiene una mirada única, unos conocimientos previos y unas formas diferentes de razonar y de aprender (Falk y Dierking, (1992) 2012; Hein, 2002). De ahí que la investigación sobre la percepción siga siendo esencial para organizar nuevas actividades educativas y formativas en los museos. 


\section{La educación museal entre la esencia y los números.}

¿Qué ha pasado durante las dos décadas que llevamos de siglo? Los museos pierden visitantes mientras que las exposiciones efímeras atraen consumidores que no llegan a entender adecuadamente la puesta en valor de las colecciones. Se han convertido para muchos en lugares de tránsito donde se mira mucho y se observa poco, y donde los interrogantes sobre la historia y la esencia de las cosas languidecen.

Esa consideración del museo como lugar de ocio, en el que se busca fidelizar para conseguir patrocinadores, hace que la misión educativa pierda significatividad. Para hacer frente a una parte de estos problemas, Eileen Hooper-Greenhill propuso ya en 1994 utilizar los planteamientos más innovadores que proceden del ámbito educativo en el museo. En realidad, se trataría de cruzar las premisas de la museología crítica con las de la pedagogía crítica para facilitar la comprensión mediante un proyecto museológico que diera sentido al espacio con relación al objeto y al contexto, y donde fuera posible el intercambio de experiencias. En esta línea encontramos la narrativa de la crítica cultural, que propone la relectura de las colecciones permanentes huyendo de lo unilateral y buscando la participación del público.

La museología crítica plantea el museo no como un dispositivo comunicador y neutro, sino que discute y cuestiona qué es cultura y patrimonio, identidad y ciudadanía. Se trata de un espacio de «[...] diálogo, conflicto, tradición, contradicción, resistencia, colisiones, funciones y transformación social.» (Alderoqui, 2011, p. 51) en el que los visitantes son comprendidos como una comunidad o redes, y los contenidos expositivos como artefactos ideológicos. Por otra parte, la pedagogía crítica es una base para que el sistema educativo fortalezca la crítica sobre la forma de construir el conocimiento y sobre el modo en que éste se convierte en fuerza social.

Padró (2015) propone entrecruzar museología y pedagogía crítica para que la función educativa del museo pueda evolucionar. Los investigadores que defienden la pedagogía crítica creen que el museo debería ser consciente de cómo se construye la diferencia. Ello los ha llevado a desarrollar métodos de interacción que - teniendo como base las teorías del conocimiento asociadas a estudiosos como Piaget, Bruner, Bloom, Ausubel, Gardner o Kolb, entre otros - han ido encontrando aplicación en el ámbito educativo de los museos. Así, han hallado una respuesta a la reflexión sobre los factores que hacen que el diseño de una exposición resulte interesante para los visitantes e incremente la participación. 
En esa búsqueda para aumentar la participación, la cuestión que debe prevalecer es cómo abordar la obra de arte en modo abiertamente inclusivo sin perder el rigor que ésta debe exigir.

\section{Construyendo la diferencia para deconstruir la desigualdad}

En torno a las narrativas museales y a los métodos de interacción que acabamos de ver, la Galleria d'Arte Moderna e Contemporanea de Bérgamo (GAMeC) utiliza desde 2007 la narración autobiográfica. Con ella, no solo da a conocer el patrimonio cultural en clave intercultural - de forma que se produzca una alfabetización crítica del espectador-narrador, active la ciudadanía y cree un sentido de «pertenencia al lugar»-, sino, fundamentalmente, como un elemento escenográfico para la interpretación de la obra de arte. Como si de un libreto se tratara, ha adoptado la narración autobiográfica para acercarse a ella, desvistiéndola del aurea y considerándola un artefacto visual, teatral, asociando el diseño y el montaje expositivo a la adecuación de la iluminación, el cuerpo y la palabra, elementos a los que recurre para componer una particular escenografía dentro del espacio museo. Ello supone un intercambio de saberes y puntos de vista. Mientras la cultura material se ofrece como metáfora para plantear la vida del objeto, de su evolución, en un contexto paralelo a la vida del intérprete-mediador museal, los significados se negocian en un juego de diálogo personal y de escucha para, en cierto modo, redefinir el sentido de la visita, concentrándolo en la fruición de cada uno de los sentidos.

\section{Huellas para la mediación cultural}

Todo inició en 2007, durante la colaboración entre el Teatro Donizetti de Bérgamo y la GAMeC en el proyecto «Tracce straniere», en el que activaron un curso de mediación museal entre el colectivo de emigrantes de Bérgamo y provincia, un capital humano para implicar a compatriotas en las visitas al museo y exposiciones mediadas en el propio idioma y cultura. El objetivo de estos encuentros consistía en utilizar el museo -lugar de encuentro e inclusiónen una plataforma de intercambio, de recíproco conocimiento y de enriquecimiento cultural. Muchos de estos mediadores han encontrado una salida profesional como guías en su lengua materna y se han convertido en piezas transmisoras de proyectos interculturales, cursos a universitarios y de actualización para docentes, así como referentes para personas con limitaciones físicas e intelectuales. 
Tras la experiencia con el Teatro Donizetti, en el año 2014, la GAMeC diseñó e implementó un proyecto al que llamaron «12 narratori in cerca d'autore», que atraviesa la colección permanente, la memoria y las tradiciones (el patrimonio cultural inmaterial) y la identidad cultural. Entendiendo que deben dar voz a narraciones diferentes, han velado la mirada del historiador de arte para que importe la del espectador/mediador, susceptible de ser representada en vivo en un espacio museístico que se transforma en espacio escénico.

Es un proyecto en el que sus creadoras -Silvia Mascheroni, Simona Bodo y Maria Grazia Panigada - velan por que el contenido que surge en torno a la lectura transversal, que el mediador hace de la obra de arte al entrelazarla con la vida cotidiana, no la corrompa (Panigada, comunicación personal, 15 febrero 2017). De este modo, el museo se convierte en un lugar para reflexionar sobre todo tipo de patrimonios, haciendo posible los derechos culturales:

«Todos tenemos un patrimonio personal, un cúmulo de vivencias que han ido marcando nuestra vida y que guardamos en forma de objetos, de recuerdos o de sus representaciones [...] Un pequeño acervo que cuando se comparte en sus significados con el grupo humano de la familia configura el patrimonio familiar. Es por ello que enfatizo la idea de "compartir significados" porque no es patrimonio cultural la mera acumulación de los bienes patrimoniales de cada uno de los miembros que constituyen una familia, sino aquel conjunto de bienes con el que la colectiva familia se identifica». (Aguirre, 2008, citado en Fernández y Gutiérrez, 2018, p.12).

\section{Compartir experiencias, hacer museo}

La narración surge por la misma necesidad o deseo de compartir una experiencia vivida o percibida. Cuando se trata de una narración autobiográfica del mediador museal, el objeto se funde con su vida, dando lugar a un diálogo en una «triangulación entre obra, narrador y visitante» (Mascheroni, Bodo y Panigada, 2016, p. 68). Esta experiencia emocional que desvela recuerdos que nacen y pertenecen a otros contextos, facilita la interpretación de las obras por esa «capacidad de transformar que hay implícita en la narración de los mediadores museales» (Fernández y Gutiérrez, 2018, p. 12). Para llegar a esa triangulación, debe existir un tiempo de escucha y de comprensión en un laboratorio narrativo, donde se entra en un espacio individual para convertirlo en otro colectivo. Una conversión que se produce cuando se cruzan características comunes que trascienden un lugar para adentrarse en otro usando el tiempo y el espacio -elementos que estructuran y expresan el lenguaje expositivo- y la acción física y/o verbal mediante recursos expresivos y emocionales. Tiempo, espacio y 
acción, al discurrir de un lugar a otro atravesando distintos conflictos y emociones, trasladan al espectador desde la historia de la representación pictórica a la experiencia humana, contribuyendo a incrementar el clima expresivo.

M.G. Panigada sostiene que la narración es el tiempo que transcurre desde que un hecho sucede, lo elaboramos y lo contamos. Su función como asesora teatral en el museo consiste en dar valor a ese tiempo y convertirlo en un relato que destile la necesidad de contarse con relación a la obra. Esto es lo más difícil, porque no siempre el narrador se fía de la verosimilitud entre lo que siente, lo que cuenta y cómo lo hace. Por ello, es muy importante la escucha, la de sí mismo y la del otro, para que se produzca un intercambio. El trabajo en talleres de la propia percepción que tienen de sí al ponerse en juego delante de los demás, facilita el acercamiento emocional, (Panigada, comunicación personal, 15 de febrero de 2017) incluido como habilidad de la inteligencia enunciada en el modelo de Mayer y Salovey (1997), que se asienta en la percepción y la comprensión. Esto ha permitido a las autoras del proyecto no solo conocer y comprender la especificidad expresiva que custodia cada una de las historias de vida, sino experimentar un método narrativo que se aleja del tradicional.

En este método hay otros objetivos: experimentar nuevos modos de presentar las obras de arte y cohesionar los distintos grupos de mediadores culturales. En este sentido, es fundamental la presencia de Simona Bodo y Silvia Mascheroni, que, como expertas en educación al patrimonio en un código intercultural, equilibran el proyecto de mediación de la colección desde los primeros momentos de acercamiento a la obra hasta su comunicación en una exposición final.

En el momento de la elección de la obra, Panigada acompaña al mediador/intérprete/narrador durante este proceso. Se sitúa delante de la obra hasta que se vuelva familiar, por eso pide una reflexión previa ante su elección: ¿Debe recaer sobre aquella que mejor conoce y está más expuesta durante las visitas guiadas? ¿Intenta evitar las más simbólicas del museo porque se arriesgaría a emitir un juicio superficial? Ambos criterios desvían de la intención inicial, porque, siguiendo uno u otro, es difícil entrelazar la obra con el elemento autobiográfico o se realiza de forma forzada (Bodo et al, 2016, p. 62). 


\section{Evocar el objeto para que aparezca la obra.}

A veces el mismo mediador cambia la obra elegida, incluso muy «respirada». Y es que, como apunta Panigada, el mismo mediador percibe que el resultado no se acerca a una narración autobiográfica porque se convierte en algo demasiado racional y poco creíble. A menudo la elección no es casual, pero cuando la elección es inmediata por la capacidad evocadora del objeto, el elemento autobiográfico se funde con la historia artística de la obra, produciéndose un cruce entre el significado de la exposición y el contexto de cada historia de vida, adquiriendo significados diferentes en uno y otro sentido. Sea como fuere, la relación entre el mediador y la obra elegida es profunda, bastante más de lo que parece al principio, experimentando una sensación especial cuando se produce la inmersión en el objeto escogido.

Cada persona tiene un modo diferente de narrar su historia, y esta tiene que estar bien contada no solo para hacerla interesante a los ojos de potenciales espectadores, sino para desprenderse de los referentes que haya podido ir acumulando en torno a la obra a lo largo de su vida. La historiadora de arte proporciona (siempre a la luz de últimas investigaciones) material de lectura para que nada quede fuera desde el punto de vista histórico-artístico. Esto no quiere decir que exista manipulación porque, la lectura de las obras elegidas con perspectivas diferentes cuyo fin es que afloren las emociones de la experiencia humana, se trata siempre de un trabajo de búsqueda individual, pero no solo autorreferencial, sino $[\ldots]$ «fortemente intrecciata alla storia dell'opera e di chi l'ha creata, al suo posizionamento nella storia dell'arte, con riferimenti all'epoca in cui è stata realizzata, al contesto storico ed artístico che ha contribuito a produrla» (Bodo et al, 2016, p.60). «Hacer creíble cada narración en su diálogo con el museo» (Fernández y Gutiérrez, 2018, p. 13), es lo que Maria Grazia Panigada, y Emanuela Daffra con Paola Strada como historiadoras del arte, tratan de conseguir,

El mediador-narrador entra en la obra y la interpreta utilizando (a modo de instrumentos escenográficos) el cuerpo, la palabra y las cualidades de la voz, entrecruzadas unas veces con la sonoridad de la propia lengua y otras con un idioma adoptado, dulcificado desde el propio acento. A ello se une la escenografía particular de un juego lumínico que destaca la sinergia entre la obra y quien la interpreta, potenciado por las sombras generadas con la disposición de un público que, a su vez, interactúa e interacciona con el narrador y con la obra de arte. 


\section{Conclusiones. Finalidad y puntos críticos}

Como en todo proyecto educativo, existe una serie de puntos críticos que hay que considerar, centrados fundamentalmente en el modo de comprender y vivir la relación con la obra de arte. Los comisarios o curadores ven el acercamiento narrativo muy alejado del contexto etnográfico o arqueológico del objeto, encontrando dificultad para reconocer en los contenidos creador conexiones en su representación y presentación al público. A pesar de que la institución busca en todo momento mantener el rigor científico, no se ha librado de las críticas de algunos de ellos, que sienten cierta repulsa e incomodidad ante demasiada subjetividad en la forma de abordar la comprensión de las obras, aunque en otras ocasiones ha sido acogida y aceptada al reconocer las competencias narrativas del interlocutor.

Aun así, no hay que olvidar que la finalidad primordial de este proyecto biográficonarrativo - ideado por la Galleria d'Arte Moderna y Contemporanea de Bérgamo y adoptado en otros museos italianos -, es hallar una herramienta que acerque el arte a aquellos colectivos que desconocen los códigos que les permitan descifrar e interpretar algunas obras de arte. Se trata de un instrumento inclusivo en el proceso de patrimonialización de una cultura que, la mayoría de las veces les es completamente ajena. Para compartir la propia cultura, es insustituible la comprensión y el entendimiento, por ello, la narración, es un gesto que implica aprender la actitud hacia la escucha y la mirada. Se trata, en definitiva, de un modo de acercar el arte a lo universal desde lo individual, una acertada defensa de los derechos culturales partiendo del patrimonio cultural.

\section{Referencias bibliográficas}

Aguirre, Imanol (2008). "Nuevas ideas de arte y cultura para nuevas perspectivas en la difusión del patrimonio", en Imanol Aguirre, Olaia Fontal, Bernard Darras \& René Rickenmann (Coords.) El acceso al patrimonio cultural: Retos y Debates. Cuadernos de la Cátedra Jorge Ateiza, Universidad Pública de Navarra (UPNA): Pamplona, pp. 67-118.

Alderoqui, Silvia (2011). La educación en los museos: de los objetos a los visitantes, Paidós: Argentina. Falk, John Howard \& Dierking, Lynn Diane (2012). The museum experience. Lef Coast Press: California. 
Fernández-Inglés S. \& Gutiérrez-Pérez, R. (2018). El método biográfico narrativo aplicado a los bienes culturales. Una experiencia inclusiva e intercultural en la GAMeC. Tercio Creciente, 14, págs. 7-16. https://dx.doi.org/10.17561/rtc.n14.1

Hein, George E. (2002). Learning in the Museum. Routledge: London \&NY.

Hooper-Greenhill, Eileen (2007). Museums and Education. Purpose, pedagogy, performance. Routledge: London \&NY.

Mayer, John \& Salovey, Peter. (1997). “What is emotional intelligence?” En Peter Salovey \& David Sluyter (Eds). Emotional Development and Emotional Intelligence: Implications for Educators (pp. 3-31). New York: Basic Books, pp.3-31

Padró, Carla (2005). "Educación artística en museos y centros de arte", en Ricard Huerta \& Román de la Calle (Eds). La mirada inquieta. Educación artística y museos. Universidad Politécnica de Valencia (UPV): Valencia, pp. 137-152.

Panigaada, Maria Grazia (2016). "Esperimenti narrativi in museo", in Simona Bodo, Silvia Mascheroni \& Maria Grazia Panigada (eds). Un patrimonio di storie. La narrazione nei musei, una risorsa per la cittadinanza culturale, Mimesis: Milano, pp. 51-69.

Zeller, Terry (1989). "The Historical and Philosophical Foundations of Art Museum Education", in Nancy Berry \& Susan Mayer (eds.) Museum Education: History, Theory and Practice. National Art Education Association: Reston, Virginia, pp. 10-89. 